\title{
A search for pulsating blue stars in the vicinity of NGC 6791 using Kepler LC data
}

\author{
Paulina Sowicka ${ }^{1, a}$, Szymon Bachulski ${ }^{2}$, and Andrzej Baran ${ }^{2}$ \\ 1 Nicolaus Copernicus Astronomical Center, Bartycka 18, 00-716 Warsaw, Poland \\ 2 Pedagogical University, Podchorążych 2, 30-084 Cracow, Poland
}

\begin{abstract}
There are 18 known pulsating subdwarf B stars in the field of view of the Kepler spacecraft during phase one. The majority of them were observed in the short cadence mode and have been successfully investigated via asteroseismic methods. Since these stars are important for the stellar evolution theory, we performed a search for blue stars in the vicinity of the open cluster NGC 6791, which was observed by the Kepler in the long cadence mode in the so-called super apertures. We used Q1-Q17 LC data and performed pixel analysis using PyKE and our customized scripts. We preliminary tagged 23 objects and we calculated amplitude spectra to look for periodic signal which exist in the data. We found four stars with significant signal in the amplitude spectra with none of them classified as gravity modes in pulsating hot subdwarfs. Likely, all four objects are binaries, though, spectroscopic observations are needed to sort out our hypothesis.
\end{abstract}

\section{Introduction}

Hot subdwarf B stars are 0.5 solar masses stars with effective temperatures from 20000 to $40000 \mathrm{~K}$. They are located at the blue end of the horizontal branch, burning helium in their cores. During their evolution on a red giant stage they have lost all but a tiny part of the hydrogen envelopes, therefore subdwarf B stars will not climb up to the asymptotic branch, moving directly to the white dwarf cooling track, instead.

The photometric data collected by the Kepler spacecraft are continuous and of unprecedented quality. They allowed us to detect equal period spacing sequences and multiplets in many pulsating subdwarf B stars. These two properties are very powerful tools for mode identification, which is a necessary step in the application of asteroseismology to study stellar interiors. Encouraged by this phenomenal achievement we looked for more pulsating sdB stars in the vicinity of the open cluster NGC 6791, located in the Kepler field of view.

\section{Method}

We used DSS images available at wikisky.org. First, we visually inspected the cluster and its surroundings to look for blue-ish objects which represent hot members. We do not have spectral classifications of these stars, though, should any shows a significant periodic signal in the data, the object would hop onto the list of targets for further spectroscopic observations. Preliminary we tagged 23 objects and we downloaded LC data from the MAST database. Since these were super-apertures we only had pixel tables to our disposal. We used PyKE and our scripts to detrend and clip data. We applied 90-day bins for detrending and $4 \sigma$ clipping. Finally, we calculated amplitude spectra between 0 and $24 \mathrm{c} / \mathrm{d}$ (Nyquist frequency of LC data) to detect significant signal. The detection threshold was adopted at

a e-mail: paula@camk.edu.pl

This is an Open Access article distributed under the terms of the Creative Commons Attribution License 4.0, which permits unrestricted use, distribution, and reproduction in any medium, provided the original work is properly cited. 

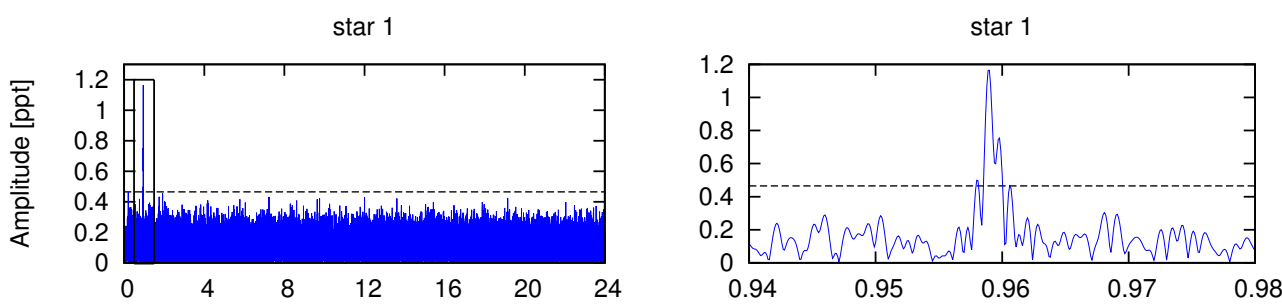

star 2
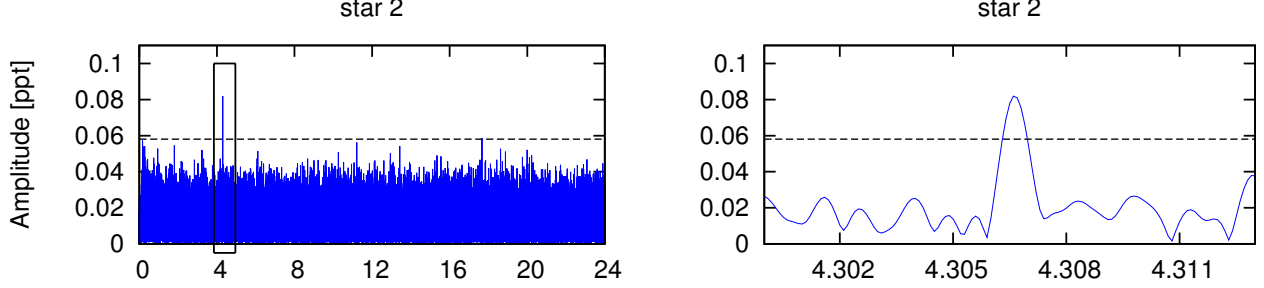

star 3
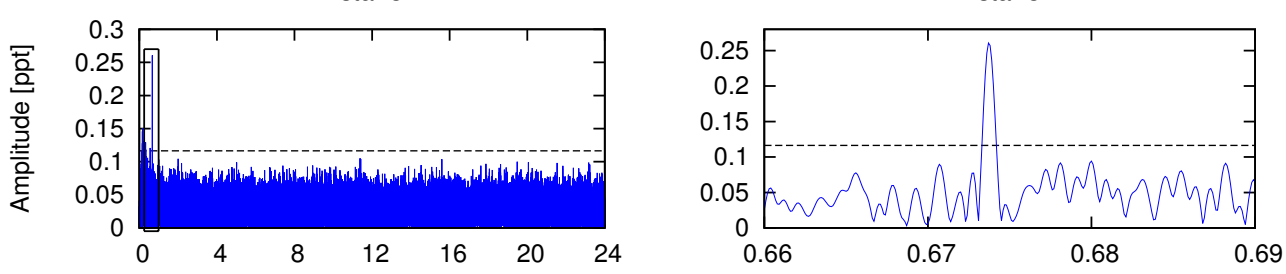

star 4

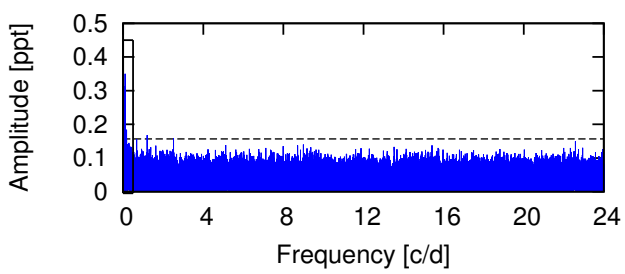

star 4

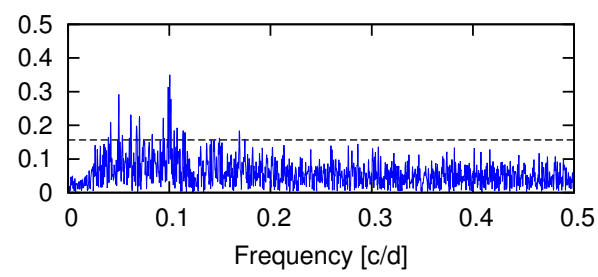

Fig. 1. Left panels show the amplitude spectra calculated all the way up to the Nyquist frequency. Right panels present close-ups of amplitude spectra to show details of the significant signal. The horizontal dashed lines represent $4 \sigma$ detection threshold.

4 times the average noise level in the amplitude spectrum. Only four objects show any evidence of significant signal in the photometric data. The periods are rather long for gravity modes in the subdwarf B stars, hence we expect that they are either binaries or other types of pulsating stars which show long period gravity modes, e.g. slowly pulsating B stars. We show our results in Fig.1.

\section{Future prospects}

Since we do not have spectral classifications of those four targets in our possession, we are unable to conclude definitively on the variability types. We have already undertook an effort to make spectroscopic observations to investigate the types of variability we detected. Our success depends on the telescope allocation and we keep our fingers crossed that the spectra will be available to us soon. 